\title{
THE COEFFICIENT PROBLEM OF REGULAR FUNCTIONS
}

\author{
JUN-ITI ITÔ
}

1. Introduction. Let $f(z)$ be a function meromorphic on $0 \leqq \rho \leqq|z|$ $\leqq 1$. Let $R f(z)$ be non-negative or change sign a finite number of times on $|z|=1$ except for poles. Then the coefficients of the Laurent expansion of $f(z)$ in $\rho^{\prime}<|z|<1$ have been studied in a previous paper $[3$, Theorem 2].

In this note we shall study the coefficients under weaker conditions than those of the paper cited above.

2. Preliminaries. Let $\sigma_{k}, k=1, \cdots, 2 s$, denote $2 s$ real numbers such that $\left|\sigma_{k}\right| \leqq \pi, k=1, \cdots, 2 s$ and set

$$
g(s, z)=K z^{-s} \prod_{k=1}^{2 s}\left(e^{i \sigma_{k}}-z\right)
$$

where $K$ is a constant of modulus unity. Then we may give two definitions.

Definition 1. A function $f(z)$ regular in $0 \leqq \rho \leqq|z|<1$ is said to belong to a class $B(S, M)$, if

$$
\int_{-\pi}^{+\pi}\left|R T\left(r e^{i \phi}\right)\right| d \phi<c<\infty
$$

for $\rho<r<1$, where $T(z)=f(z) g(s, z)$ and if, for a non-negative constant $M$,

$$
\lim _{r \rightarrow 1} \int_{-\pi}^{+\pi}\left[R T\left(r e^{i \phi}\right)\right]^{-} d \phi \leqq 2 \pi M
$$

where $[R T(z)]^{-}=\operatorname{Max}\{0,-R T(z)\}$.

Definition 2. A function $f(z)$ regular in $0 \leqq \rho \leqq|z|<1$ is said to belong to a class $B^{\prime}(S, M)$ if $g(s, z)$ satisfies the following: (a) both 0 and $\pi$ are members of the set $\sigma_{k}, k=1, \cdots, 2 s$; (b) if $e^{i \sigma_{k}}$ for $k=1,2, \cdots, 2 s\left(\sigma_{k} \neq 0, \pi\right)$ is an $l$ th order zero, then $e^{-i \sigma_{k}}$ is also an $l$ th order zero, $l$ being a positive integer, and if $f(z)$ is a member of the class $B(S, M)$.

LemMa. Let $u(z)$ be harmonic in $|z|<1$. Suppose that $\lim _{\text {inf }} z_{\rightarrow z^{\prime}} u(z)$ $\geqq 0$ for every boundary point $z^{\prime}$ except for a finite number of boundary

Received by the editors December 8, 1959 and, in revised form, February 19, 1960. 
points $z_{1}, \cdots, z_{m}$. Let $u(z)=O\left(\left|z-z_{k}\right|^{-1}\right)$ as $z \rightarrow z_{k}, k=1, \cdots, m$. Then

$$
\lim _{r \rightarrow 1} \int_{-\pi}^{+\pi}\left|u\left(r e^{i \phi}\right)\right| d \phi<k<\infty .
$$

This lemma has been proved in [3, Lemma 4].

3. Theorems. If we use notations due to Goodman and Robertson

$$
\begin{aligned}
& \Delta(s, k, n)=2 \prod_{v(\neq k)=0}^{s}\left(n^{2}-v^{2}\right) /(s+k) !(s-k) ! \quad(s \geqq k>0), \\
& \Delta(s, 0, n)=\prod_{v=1}^{s}\left(n^{2}-v^{2}\right) /(s !)^{2}, \\
& \text { (3.2) } D(s, k, n)=2 k(n+s) ! /(s+k) !(s-k) !(n-s-1) !\left(n^{2}-k^{2}\right) \\
& (s \geqq k>0) \text {, }
\end{aligned}
$$

we obtain the following main Theorems 1 and 2 .

THEOREM 1. Let

$$
f(z)=\sum_{n=-\infty}^{\infty} a_{n} z^{n}
$$

be regular in $0 \leqq \rho \leqq|z|<1$ and be a member of the class $B(S, M)$. Then for all $n>s$, the following inequalities hold

$$
\begin{aligned}
\left|a_{n}+\delta \bar{a}_{-n}\right| \leqq & \sum_{k=0}^{s-1} \Delta(s, k, n)\left|a_{k}+\delta \bar{a}_{-k}\right| \\
& +\Delta(s, s, n)\left(\left|a_{s}+\delta\left(\bar{a}_{-s}+K^{2} M\right)\right|+M\right)
\end{aligned}
$$

where $\delta=\bar{K}^{2} e^{-i} \sum_{k=1}^{2 s} \sigma_{k}$. In case $M=0$ and $\sigma_{k}=0, k=1,2, \cdots, 2 s$, these equality signs hold.

A theorem of Robertson [5, Theorem 1] in which the imaginary part of $f(z)$ changes the sign $2 P$ times is obtained by putting $M=0$ and $\delta=1$.

Proof. Set

$$
e^{i \lambda / 2}=K \exp \left(\frac{i}{2} \sum_{k=1}^{2 s} \sigma_{k}\right), \quad f_{1}(z)=\sum_{n=-\infty}^{\infty} e^{i \lambda / 2} a_{n} z^{n}=\sum_{n=-\infty}^{\infty} a_{n}^{\prime} z^{n}
$$

and

$$
G(z)=f_{1}(z)-\sum_{n=1}^{\infty}\left(a_{-n}^{\prime} z^{-n}-\bar{a}_{-n}^{\prime} z^{n}\right)
$$


Then $G(z)$ is regular in $|z|<1$. The series of the right side of (3.5) is regular and its real part is identically zero on $|z|=1$. If we put

$$
F(z)=e^{-i \lambda / 2} g(s, z) G(z)=\sum_{n=-8}^{\infty} A_{n} z^{n}
$$

and

$$
E(z)=F(z)-\sum_{n=1}^{8}\left(A_{-n} z^{-n}-\bar{A}_{-n} z^{n}\right),
$$

then $E(z)$ is regular in $|z|<1$ and for $\rho<r<1$

$$
\begin{aligned}
& \int_{-\pi}^{+\pi}\left|R E\left(r e^{i \phi}\right)\right| d \phi=\int_{-\pi}^{+\pi}\left|R F\left(r e^{i \phi}\right)\right| d \phi \\
&=\int_{-\pi}^{+\pi} \mid R T\left(r e^{i \phi}\right)-R\left\{\sum_{n=1}^{\infty}\left(a_{-n}^{\prime}\left(r e^{i \phi}\right)^{-n}-\bar{a}_{-n}^{\prime}\left(r e^{i \phi}\right)\right)\right. \\
&\left.\cdot \frac{1}{K} \exp \left(-\frac{i}{2} \sum_{k=1}^{2 s} \sigma_{k}\right) g\left(s, r e^{i \phi}\right)\right\} \mid d \phi .
\end{aligned}
$$

Therefore, from the hypothesis the integral of the left side of (3.8) is bounded for $\rho<r<1$. Hence $R E(z)$ is represented as the difference of two non-negative harmonic functions in $|z|<1$. From Poisson's representation theorem for the non-negative harmonic function, we get the relation of (2.4) for $E(z)$. By F. Nevanlinna's theorem [4], the limit $\alpha(\theta)=\lim _{r \rightarrow 1} \int_{-\pi}^{\theta} R E\left(r e^{i \phi}\right) d \phi,|\theta| \leqq \pi$, exists except for at most a countable set of $\theta$. The function $\alpha(\theta)$ is of bounded variation and $E(z)$ is represented as a Stieltjes' integral $(1 / 2 \pi) \int_{-\pi}^{+\pi}\left(\left(e^{i \theta}+z\right) /\left(e^{i \theta}\right.\right.$ $-z)) d \alpha(\theta)+i I E(0)$. Obviously $\alpha(\theta),|\theta| \leqq \pi$, is a difference of two monotone increasing functions $\alpha_{1}(\theta)$ and $\alpha_{2}(\theta)$, that is, $\alpha(\theta)=\alpha_{1}(\theta)-\alpha_{2}(\theta)$ where $\alpha(-\pi)=\alpha_{1}(-\pi)=\alpha_{2}(-\pi)=0$ and the total variation of $\alpha$ in $(-\pi, \theta)$ is equal to $\alpha_{1}+\alpha_{2}$. Moreover

$$
\begin{aligned}
\alpha_{2}(\pi) & =\lim _{r \rightarrow 1} \frac{1}{2} \int_{-\pi}^{+\pi}\left\{\left|R E\left(r e^{i \phi}\right)\right|-R E\left(r e^{i \phi}\right)\right\} d \phi \\
& =\lim _{r \rightarrow 1} \int_{-\pi}^{+\pi}\left[R E\left(r e^{i \phi}\right)\right]-d \phi \\
& =\lim _{r \rightarrow 1} \int_{-\pi}^{+\pi}\left[R T\left(r e^{i \phi}\right)\right]-d \phi \leqq 2 \pi M .
\end{aligned}
$$

Therefore $E(z)$ is a member of the class $B(0, M)$. Set 


$$
L(z)=E(z)+\frac{1}{2 \pi} \int_{-\pi}^{+\pi} \frac{e^{i \theta}+z}{e^{i \theta}-z} d \alpha_{2}(\theta)
$$

then $R L(z) \geqq 0$ for $|z|<1$. By using the results of Robertson [5] and the author [3], we find that for $n>s$

$$
\begin{gathered}
G(z)+\frac{e^{i \lambda / 2}}{2 \pi} \int_{-\pi}^{+\pi} \frac{e^{i \theta}+z}{\left(e^{i \theta}-z\right) g(s, z)} d \alpha_{2}(\theta)=\sum_{n=0}^{\infty} B_{n} z^{n} \\
\ll \sum_{n=8+1}^{\infty}\left(\sum_{k=0}^{s-1} \Delta(s, k, n)\left|a_{k}+e^{-i \lambda} \bar{a}_{-k}\right|\right. \\
\left.\quad+\Delta(s, s, n)\left|a_{s}+e^{-i \lambda}\left(\bar{a}_{-s}+K^{2} M\right)\right|\right) z^{n} .
\end{gathered}
$$

Moreover, we get

$$
\frac{1}{2 \pi} \int_{-\pi}^{+\pi} \frac{e^{i \theta}+z}{\left(e^{i \theta}-z\right) g(s, z)} d \alpha_{2}(\theta) \ll \sum_{n=s+1}^{\infty} \Delta(s, s, n) M z^{n} .
$$

Thus from (3.11) and (3.12) we get (3.4). The majorant series of (3.11) is attained if $\sigma_{k}=0, k=1, \cdots, 2 s$.

CoRollary 1. Let $f(z)$ of the form (3.3) be regular in $0 \leqq \rho \leqq|z|<1$. Suppose that lim inf $R\{\gamma f(z)\} \geqq 0$ for every boundary point except for a finite number of points $z_{1}, \cdots, z_{m}$ on $|z|=1$ where $|\gamma|=1$. Let $\tau_{k}, k=1, \cdots, m$, be non-negative numbers, $R\{\gamma f(z)\}=O\left(\left|z-z_{k}\right|-\tau_{k}\right)$, $k=1, \cdots, m$ and $l_{k}=\left[\tau_{k} / 2\right], k=1, \cdots, m$. Finally let for $t$ points $z_{m_{v}}=e^{i \theta m_{v}}, v=1, \cdots, t$, among $z_{k}, k=1, \cdots, m$,

$$
\lim _{r \rightarrow 1}(-1)^{l m_{v}} \gamma \prod_{k\left(\neq m_{v}\right)=1}^{m}\left|z_{k}-z_{m_{v}}\right| 2 l_{k}(1-r)^{2 l m_{v}+1} R f\left(r e^{i \theta m_{v}}\right)=\mu_{m_{v}}<0
$$

and let the above limits be non-negative for the other $m-t$ points among $z_{k}, k=1, \cdots, m$. Then for $n>s=2 \sum_{k=1}^{m} l_{k}$

$$
\begin{aligned}
\left|a_{n}+\bar{r}^{2} \bar{a}_{-n}\right| \leqq & \sum_{k=0}^{s} \Delta(s, k, n)\left|a_{k}+\bar{r}^{2} \bar{a}_{-k}\right| \\
& -2 \Delta(s, s, n) \sum_{v=1}^{t} \mu_{m_{v} .}
\end{aligned}
$$

If $t=0$, these bounds are sharp.

Proof. By the hypothesis, we may take

$$
g(s, z)=\prod_{k=1}^{m}\left\{\left(z_{k}-z\right)\left(\bar{z}_{k}-\frac{1}{z}\right)\right\}^{l_{k}} \text {. }
$$


Therefore, putting $F(z) g(s, z) G(z)=\sum_{n=-s}^{\infty} A_{n} z^{n}$ for $G(z)$ of (3.5) with $e^{i(\lambda / 2)}=\gamma, F(z)$ satisfies the hypothesis of the lemma. Hence using a theorem by H. Herzig [2] for $E(z)$ of the form (3.7), we find that for $z_{m_{v}}, v=1, \cdots, t$ and $\alpha_{2}(\theta)$ of $\S 3$,

$$
\lim _{r \rightarrow 1}(1-r) R E\left(r e^{i \theta m_{v}}\right)
$$

$$
=\frac{1}{2 \pi}\left(\alpha_{2}\left(\theta_{m_{v}}+0\right)-\alpha_{2}\left(\theta_{m_{v}}-0\right)\right)=\mu m_{v}<0
$$

and for the other $m-t$ points among $z_{k}, k=1, \cdots, m$, the above limits are non-negative. Therefore from Theorem 1 we get (3.13). And the bounds of (3.13) are sharp if $t=0$.

THEOREM 2. Let

$$
f(z)=\sum_{n=1}^{\infty} a_{n} z^{n}
$$

be regular in $|z|<1$. Suppose that $f(z)$ is a member of the class $B^{\prime}(S, M)$ and that $K a_{k}, k=1, \cdots, s-1$, are imaginary. Then for $n>s$

$$
\left|a_{n}\right| \leqq \sum_{k=1}^{s} D(s, k, n)\left|a_{k}\right|+2 D(s, s, n) M .
$$

If $M=0$, these bounds are sharp.

A theorem of Goodman and Robertson [1] on the class $T(P)$ is obtained by putting $M=0$.

Proof. Let $g(s, z)$ be a function defined in Definition 2. Set

$$
F(z)=g(s, z) \sum_{n=1}^{\infty} a_{n} z^{n}=\sum_{n=-s+1}^{\infty} A_{n} z^{n}
$$

Moreover, set

$$
E(z)=F(z)-\sum_{n=1}^{8-1}\left(A_{-n} z^{-n}-\bar{A}_{-n} z^{n}\right)
$$

Then by the argument used in the proof of Theorem 1, we have $E(z) \in B^{\prime}(0, M)$. Using $\alpha_{2}(\theta)$ in the proof of Theorem 1 , we can write

$$
L(z)=E(z)+\frac{1}{2 \pi} \int_{-\pi}^{+\pi} \frac{e^{i \theta}+z}{e^{i \theta}-z} d \alpha_{2}(\theta) .
$$

Obviously $R L(z) \geqq 0$ for $|z|<1$. Hence from the hypothesis that $K a_{k}$, 
$k=1, \cdots, s-1$, are imaginary and the hypothesis for $g(s, z)$, follows that for $n>s$

$$
\begin{aligned}
f(z) & +\frac{1}{2 \pi} \int_{-\pi}^{+\pi} \frac{e^{i \theta}+z}{\left(e^{i \theta}-z\right) g(s, z)} d \alpha_{2}(\theta)=\sum_{n=1}^{\infty} B_{n} z^{n} \\
& \ll \sum_{n=8+1}^{\infty}\left(\sum_{k=1}^{s} D(s, k, n)\left|a_{k}\right|+D(s, s, n)\left|K a_{s}-M\right|\right) z^{n}
\end{aligned}
$$

where $M=(1 / 2 \pi) \int_{-\pi}^{+\pi} d \alpha_{2}(\theta)$ if we apply the argument due to Goodman and Robertson [1] and the author [3]. Thus for $n>s$

$$
f(z) \ll \sum_{n=s+1}^{\infty}\left(\sum_{k=1}^{s} D(s, k, n)\left|a_{k}\right|+2 D(s, s, n) M\right) z^{n} .
$$

Hence we get (3.17). The majorant series of (3.20) is sharp. Therefore, if $M=0$, the bounds of (3.17) are sharp.

CoRollary 2. Let $f(z)$ of the form (3.16) be regular in $|z|<1$ and let $f(z) g(s, z)$ satisfy the conditions of the lemma with $g(s, z)$ as in Definition 2. Suppose that for $t$ points $z_{k}=e^{i \theta_{k}}, k=1, \cdots, t$, $\lim _{r \rightarrow 1}(1-r) g\left(s, r e^{i \theta_{k}}\right) f\left(r e^{i \theta_{k}}\right)=\mu_{k}<0$ and for the other $m-t$ points among $z_{k}, k=1, \cdots, m$, the above limits are non-negative and that $K a_{k}, k=1$, $\cdots, s-1$, are imaginary. Then for $n>s$

$$
\left|a_{n}\right| \leqq \sum_{k=1}^{s} D(s, k, n)\left|a_{k}\right|-2 D(s, s, n) \sum_{v=1}^{t} \mu_{m_{v}} .
$$

If $t=0$, these bounds are sharp.

The proof of this corollary is obtained by the same method as in the proof of Corollary 1.

Two theorems of Robertson and Goodman [1, Theorem 4] and Robertson [5, Theorem 2] are generalized by the following Theorems 4 and 3 respectively.

TheOREM 3. Let $f(z)$ of the form (3.3) be regular in $0 \leqq \rho \leqq|z|<1$ and let $z(d f(z) / d z) \in B(S, M)$. Then for $n>s$

$$
\left|a_{n}+\delta \bar{a}_{-n}\right| \leqq \sum_{k=1}^{s} D(s, k, n)\left|a_{k}+\delta \bar{a}_{-k}\right|+(2 / n) \Delta(s, s, n) M,
$$

where $\delta=\bar{K}^{2} \exp \left[-i\left(\sum_{k=1}^{2 s} \sigma_{k}-\pi\right)\right]$. If $M=0$ and $\sigma_{k}=0, k=1, \cdots$, $2 s$, these equality signs hold.

Theorem 4. Let $f(z)$ of the form (3.3) be regular in $0 \leqq \rho \leqq|z|<1$ and let $z d f(z) / d z \in B^{\prime}(S, M)$. Suppose that $K a_{k}+\bar{K} \bar{a}_{-k}, k=1, \cdots$, $s-1$, are imaginary. Then for $n>s$ 


$$
\begin{aligned}
& \left|a_{n}+\bar{K}^{2} \bar{a}_{-n}\right| \\
& \quad \leqq \sum_{k=1}^{s} \frac{k}{n} D(s, k, n)\left|a_{k}+\bar{K}^{2} \bar{a}_{-k}\right|+\frac{2}{n} D(s, s, n) M .
\end{aligned}
$$

If $M=0$, then these bounds are sharp.

The proofs of Theorems 3 and 4 are easily obtained.

THEOREM 5. Let

$$
f(z)=\sum_{n=0}^{\infty} a_{n} z^{n}
$$

and $h(z)=\sum_{n=0}^{\infty} d_{n} z^{n}, d_{0} \neq 0$, be regular in $|z|<1$, and $h(z) \in B(S, M)$. Let $f(z) / h(z)$ be regular in $|z|<1$ and be a member of the class $B\left(0, M^{\prime}\right)$. Then for $n>s$

$$
\begin{aligned}
\left|a_{n}\right| \leqq & \left\{2 \sum_{l=s}^{n-1}\left(\sum_{k=0}^{s} \Delta(s, k, l)\left|d_{k}\right|+2 \Delta(s, s, l) M\right)+2 \sum_{k=0}^{s-1}\left|d_{k}\right|\right. \\
& \left.+\sum_{k=0}^{s} \Delta(s, k, n)\left|d_{k}\right|+2 \Delta(s, s, n) M\right\}\left(2 M^{\prime}+\left|\frac{a_{0}}{d_{0}}\right|\right) .
\end{aligned}
$$

Proof. From the hypothesis, we find

$$
\frac{f(z)}{h(z)}=\frac{1}{2 \pi} \int_{-\pi}^{+\pi} \frac{e^{i \theta}+z}{e^{i \theta}-z} d \alpha(\theta)+i I \frac{a_{0}}{d_{0}}
$$

where $\alpha(\theta)$ is a function of bounded variation of $\theta$. Let $\alpha_{2}(\theta)$ be the function of $\S 3$. Then $(1 / 2 \pi) \int_{-\pi}^{+\pi} d \alpha_{2}(\theta)=M^{\prime}$. And

$$
\frac{f(z)}{h(z)} \ll\left(2 M^{\prime}+\left|\frac{a_{0}}{d_{0}}\right|\right) \frac{1+z}{1-z} .
$$

Thus from (3.4) and Theorem 1, we get (3.24).

Theorem 6. Let $f(z)$ of the form (3.16) be regular in $|z|<1$. Suppose that $h(z)=\sum_{n=1}^{\infty} d_{n} z^{n}, d_{1} \neq 0$, satisfies the hypothesis of Theorem 2. Let $f(z) / h(z)$ be regular in $|z|<1$ and be of the class $B\left(0, M^{\prime}\right)$. Then for $n>s$

$$
\begin{aligned}
\left|a_{n}\right|= & \left\{2 \sum_{l=s}^{n-1}\left(\sum_{k=1}^{s} D(s, k, l)\left|d_{k}\right|+2 D(s, s, l) M\right)+2 \sum_{k=1}^{s-1} d_{k}\right. \\
& \left.+\sum_{k=1}^{s} D(s, k, n)\left|d_{k}\right|+2 D(s, s, n) M\right\}\left(2 M^{\prime}+\left|\frac{a_{1}}{d_{1}}\right|\right) .
\end{aligned}
$$

The proof of this theorem is obtained by using Theorem 2 . 
4. Remark. Modifying $g(s, z)$ slightly in $\$ 1$, we get more precise versions of Theorems 1 and 2 . Let $\sigma_{k}, k=1, \cdots, 2 s$, be real numbers such that $\left|\sigma_{k}\right| \leqq \pi, k=1, \cdots, 2 s$, and set for $|K|=1$ and $p \geqq s$,

$$
g(s, p, z)=K z^{s-p} \prod_{k=1}^{p}\left(e^{i \sigma_{k}}-z\right) / \prod_{k=p+1}^{2 s}\left(e^{i \sigma_{k}}-z\right) .
$$

Then we can define the following two classes.

Definition 3. A function $f(z)$ regular in $0 \leqq \rho \leqq|z|<1$ is said to belong to a class $B(S, P, M)$ if the relation (2.2) and (2.3) hold when $g(s, z)$ is replaced by $g(s, p, z)$.

Definition 4. A function $f(z)$ regular in $0 \leqq \rho \leqq|z|<1$ is said to belong to a class $B^{\prime}(S, P, M)$ if $g(s, p, z)$ satisfies the following: $\left(\mathrm{a}^{\prime}\right)$ both 0 and $\pi$ are members of the set $\sigma_{k}, k=1, \ldots, 2 s$; $\left(\mathrm{b}^{\prime}\right)$ if $e^{i \sigma_{k}}\left(\sigma_{k} \neq 0, \pi\right)$ for each $k$ is an $l$ th order zero or pole, $e^{-i \sigma_{k}}$ is also an $l$ th order zero or pole respectively, $l$ being a positive integer, and if $f(z)$ is a member of a class $B^{\prime}(S, P, M)$.

Let $f(z)$ of the form (3.3) be regular in $0 \leqq \rho \leqq|z|<1$ and let $f(z)$ be a member of the class $B(S, P, M)$. Then by the argument used in the proof of Theorem 1, the upper bounds of the moduli of $a_{n}+\delta \bar{a}_{n}$ for all $n>s$, are obtained in terms of $M$ and $\left|a_{k}+\delta \bar{a}_{-k}\right|, k=0,1, \cdots$, $p-s$. Similarly, if $f(z)$ of the form (3.16) is regular in $|z|<1$ and is a member of the class $B^{\prime}(S, P, M)$ and if $K a_{k}, k=1, \cdots, p-s-1$ are imaginary, then, by using the argument in the proof of Theorem 2, we get the upper bounds of the moduli of $a_{n}$ for all $n>s$, in terms of $M$ and $a_{k}, k=1, \cdots, p-s$ (see the author [3]).

\section{REFERENCES}

1. A. W. Goodman and M. S. Robertson, $A$ class of multivalent functions, Trans. Amer. Math. Soc. vol. 70 (1951) pp. 127-136.

2. A. Herzig, Die Winkeldervierte und des Poisson-Stieltjes-integral, Math. Z. vol. 46 (1940) pp. 129-156.

3. J. Itô, The variation of the sign of the real part of a meromorphic function on the unit circle, Trans. Amer. Math. Soc. vol. 89 (1958) pp. 60-78.

4. R. Nevanlinna, Eindeutige analytische Funktionen, Berlin, Springer, 1936.

5. M. S. Robertson, $A$ coefficient problem for functions regular in an annulus, Canad. J. Math. vol. 4 (1952) pp. 407-423.

Nagoya Institute of Technology, Nagoya, Japan 